\title{
Neutrino factory optimization for non-standard interactions
}

\section{Joachim KOPP}

Max-Planck-Institut für Kernphysik,

Postfach 103980, D-69029 Heidelberg, Germany

E-mail: jkopp@mpi-hd.mpg.de

\section{Toshihiko OTA*}

Institut für Theoretische Physik und Astrophysik

Universität Würzburg,

Am Hubland D-97074 Würzburg, Germany

E-mail: toshihiko.ota@physik.uni-wuerzburg.de

\section{Walter WINTER}

Institut für Theoretische Physik und Astrophysik

Universität Würzburg,

Am Hubland D-97074 Würzburg, Germany

E-mail: winter@physik.uni-wuerzburg.de

\begin{abstract}
We study the optimization of a neutrino factory with respect to non-standard neutral current neutrino interactions, and compare the results to those obtained for the standard optimization. We discuss the muon energy, baselines, and oscillation channels as degrees of freedom. Our conclusions are based on both analytical calculations and on a full numerical simulation of the neutrino factory setup proposed by the international design study (IDS-NF). We consider all possible nonstandard parameters, and include their complex phases. We identify the impact of the different parameters on the golden, silver, and disappearance channels. We come to the conclusion that, even in the presence of non-standard interactions, the performance of the neutrino factory hardly profits from a silver channel detector, unless the muon energy is significantly increased compared to the IDS-NF setup. Apart from the dispensable silver channel detector, we demonstrate that the IDS-NF setup is close to optimal even if non-standard interactions are considered. We find that one very long baseline is a key component in the search for non-standard interactions, in particular for $\varepsilon_{e \tau}^{m}$ and $\varepsilon_{\tau \tau}^{m}$. This presentation is based on the work Ref.[1].
\end{abstract}

10th International Workshop on Neutrino Factories, Super beams and Beta beams

June 30 - July 52008

Valencia, Spain

\footnotetext{
* Speaker.
} 
Experimental data for the last decade established the standard (three-flavour) neutrino oscillation picture and determined the oscillation parameters. Now future experiments are planed to achieve high sensitivities to the standard oscillation parameters, such as the unknown mixing angle $\theta_{13}$ and the CP violating phase $\delta_{\mathrm{CP}}$ in the lepton mixing matrix. Because of their high precision, we can also expect that the next generation experiments are highly sensitive to non-standard physics. In this presentation, we focus on non-standard neutrino interactions (NSI) which are parametrized as four-fermion interactions,

$$
\left(\bar{v}_{\beta} \gamma^{\rho} \mathrm{P}_{L} v_{\alpha}\right)\left(\bar{f} \gamma_{\rho} \mathrm{P}_{L / R} f\right)
$$

where $f$ is an electron or a first-generation quark. Such an effective interaction can be considered as a remnant of new physics at a high energy scale. The interactions Eq. (1) affect the neutrino propagation Hamiltonian as an extra matter effect potential term

$$
H=H_{\mathrm{SO}}+A_{\mathrm{CC}}\left(\begin{array}{ccc}
\varepsilon_{e e}^{m} & \varepsilon_{e \mu}^{m} & \varepsilon_{e \tau}^{m} \\
\left(\varepsilon_{e \mu}^{m}\right)^{*} & \varepsilon_{\mu \mu}^{m} & \varepsilon_{\mu \tau}^{m} \\
\left(\varepsilon_{e \tau}^{m}\right)^{*} & \left(\varepsilon_{\mu \tau}^{m}\right)^{*} & \varepsilon_{\tau \tau}^{m}
\end{array}\right),
$$

where $H_{\mathrm{SO}}$ denotes the standard propagation Hamiltonian, and $A_{\mathrm{CC}}$ is the usual matter effect potential term defined as $A_{\mathrm{CC}} \equiv \sqrt{2} G_{F} N_{e}$ ( $N_{e}$ is the electron number density in Earth matter). The current experimental bounds to these NSI are listed in Refs. [2] and [3] (see also Ref.[4]).

In Ref. [1], we discussed the optimal setups to search for NSI with a neutrino factory and reached the following conclusions:

- The golden channel with magic baseline $(7500 \mathrm{~km})$ has a good sensitivity to $\varepsilon_{e \tau}^{m}$ [5]. Although the silver channel with $L=4000$ can also contribute, the golden channel is advantageous in a statistical sense.

- The disappearance channel plays a main role to search for $\varepsilon_{\mu \tau}^{m}$ and $\varepsilon_{\tau \tau}^{m}$. It also helps resolving the correlation between two NSI parameters, e.g., $\varepsilon_{e \tau}^{m}$ and $\varepsilon_{\tau \tau}^{m}$

- The sensitivity to NSIs depends on the beam energy. However, it becomes saturated at $E_{\mu} \gtrsim$ $20 \mathrm{GeV}$.

Taking into account the results shown above, we consider the optimization of the baselines for a neutrino factory with two Golden detectors to search for the NSI. We did the numerical simulation with GLoBES software[6] to obtain quantitative results. The experimental setup is taken from the IDS-NF 1.0 configuration [7].

The sensitivity to the $\varepsilon_{e \tau}^{m}$ parameter is shown in the left panel of Fig. 1. The dashed curves indicate the optimum configuration for the standard oscillation parameters, $\theta_{13}, \mathrm{CP}$ violation, and mass hierarchy, which is $L=4000 \mathrm{~km}$ plus $7500 \mathrm{~km}$. The plot shows that the optimum region for this NSI almost coincides with that for the standard oscillation physics. Although the sensitivity somewhat depends on the choice of the simulated values of $\theta_{13}$ and $\delta_{\mathrm{CP}}$, the qualitative discussion for the optimal configuration does not change. We also studied the other NSI parameters in Ref.[1] and concluded that at least one very long baseline was an important prerequisite to obtain the high sensitivity to NSI. 
Our main results are summarized in the right panel of Fig.1. Here, the baselines were set to $L=4000+7500 \mathrm{~km}$. We also considered the silver $v_{e} \rightarrow v_{\tau}$ channel, and found a synergetic but only small contribution to the $\left|\varepsilon_{e \tau}^{m}\right|$ sensitivity if $E_{\mu} \gtrsim 25 \mathrm{GeV}$. This panel also shows that the NSI sensitivities do not significantly improve anymore as a function of the muon energy if $E_{\mu} \gtrsim 25$ $\mathrm{GeV}$. We concludes that the sensitivity to $\left|\varepsilon_{e \tau}^{m}\right|$ can reach $9.4 \cdot 10^{-3}(3 \sigma)$ with the IDS-NF baseline setup, which is an improvement on the current bound by two orders.
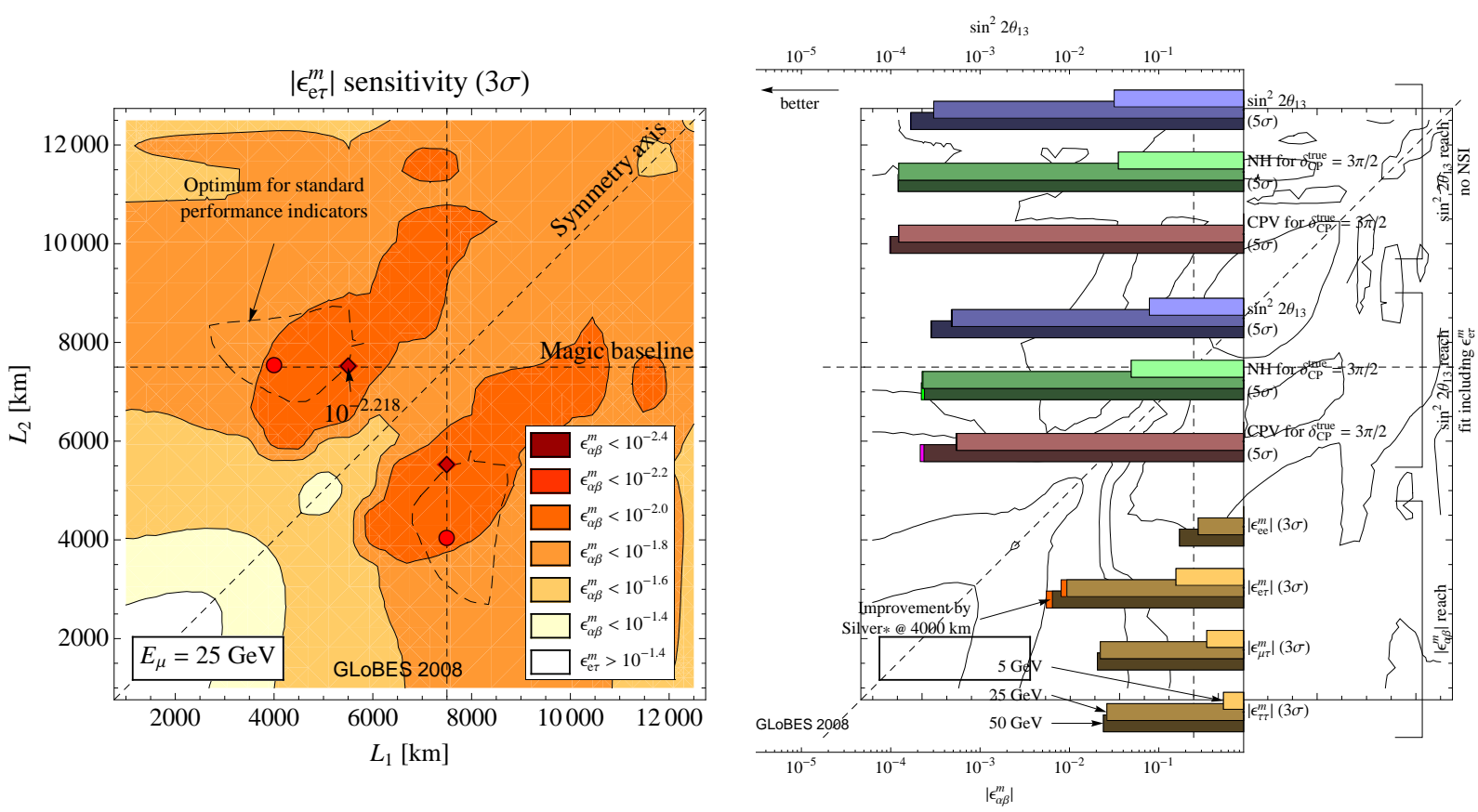

Figure 1: Sensitivity to $\varepsilon_{e \tau}^{m}$ on the two-baseline plane for two Golden detectors (left), and summary of the sensitivities to standard and non-standard parameters in the IDS-NF setup with $L=4000 \mathrm{~km}+7500 \mathrm{~km}$. Original figures appear in Ref. [1].

\section{References}

[1] J. Kopp, T. Ota, and W. Winter, arXiv:0804.2261 [hep-ph], to be published PRD.

[2] S. Davidson, C. Pena-Garay, N. Rius, and A. Santamaria, JHEP 0303011 (2003), hep-ph/0302093.

[3] M. C. Gonzalez-Garcia and M. Maltoni, Phys. Rept. 4601 (2008), arXiv:0704.1800 [hep-ph].

[4] O. Yasuda, talk at this conference.

[5] N. C. Ribeiro, H. Minakata, H. Nunokawa, S. Uchinami, and R. Zukanovich-Funchal, JHEP 0712002 (2007), arXiv:0709.1980 [hep-ph].

[6] P. Huber, J. Kopp, M. Lindner, M. Rolinec, and W. Winter, Comput. Phys. Commun. 177432 (2007), hep-ph/0701187; P. Huber, M. Lindner, and W. Winter, Comput. Phys. Commun. 167195 (2005), hep-ph/0407333; http://www.mpi-hd.mpg.de/lin/globes/

[7] ISS Physics Working Group (A. Bandyopadhyay et al.), arXiv:0710.4947 [hep-ph]; http://www.hep.ph.ic.ac.uk/ids/ 